\title{
Discovery of VVV CL001
}

\section{A low-mass globular cluster next to UKS 1 in the direction of the Galactic bulge}

\author{
D. Minniti ${ }^{1}$, M. Hempel ${ }^{1}$, I. Toledo ${ }^{1}$, V. D. Ivanov ${ }^{2}$, J. Alonso-García ${ }^{1}$, R. K. Saito ${ }^{1}$, M. Catelan ${ }^{1}$, D. Geisler ${ }^{3}$, \\ A. Jordán ${ }^{1}$, J. Borissova ${ }^{4}$, M. Zoccali ${ }^{1}$, R. Kurtev ${ }^{4}$, G. Carraro ${ }^{2}$, B. Barbuy ${ }^{5}$, J. Clariá ${ }^{6}$, \\ M. Rejkuba ${ }^{7}$, J. Emerson ${ }^{8}$, and C. Moni Bidin ${ }^{3}$ \\ 1 Departamento de Astronomía y Astrofísica, P. Universidad Católica de Chile, Casilla 306, Santiago, Chile \\ e-mail: [dante;mhempel; itoledoc; jalonso;r.saito;mcatelan;ajordan;mzoccali] @astro.puc.cl \\ 2 European Southern Observatory, Alonso de Córdova 3107, Vitacura, Casilla 19001, Santiago de Chile 19, Chile \\ e-mail: [vivanov; gcarraro] @eso.org \\ 3 Departamento de Astronomía, Universidad de Concepción, Bio-Bio 160-C, Chile \\ e-mail: [dgeisler; cmbidin] @astro-udec.cl \\ 4 Departamento de Física y Astronomía, Facultad de Ciencias, Universidad de Valparaíso, Av. Gran Bretaña 1111, Valparaíso, Chile \\ e-mail: [jura.borissova; radostin.kurtev]@uv.cl \\ 5 Inst Astronomico e Geofisico-Depto Astronomia, Universidade de São Paulo, Rua do Matão 1226, Cidade Universitária, \\ São Paulo 05508-900, Brazil \\ e-mail: barbuy@astro.iag.usp.br \\ 6 Observatorio Astronomico, Laprida 854, Córdoba 5000, Argentina \\ e-mail: jjclaria@gmail.com \\ 7 European Southern Observatory, Karl-Schwarszchild Strasse 2, 85748 Garching/ bei München, Germany \\ e-mail: mrejkuba@eso.org \\ 8 Astronomy Unit, School of Mathematical Sciences, Queen Mary, University of London, Mile End Road, London, E1 4NS, UK, \\ e-mail: j.p.emerson@qmul.ac.uk
}

Received 20 September 2010 / Accepted 28 November 2010

ABSTRACT

\begin{abstract}
Context. It is not known how many globular clusters may remain undetected towards the Galactic bulge.
Aims. One of the aims of the VISTA Variables in the Via Lactea (VVV) Survey is to accurately measure the physical parameters of the known globular clusters in the inner regions of the Milky Way and search for new ones, hidden in regions of large extinction. Methods. From deep near-infrared images, we derive deep $J H K_{\mathrm{S}}$-band photometry of a region surrounding the known globular cluster UKS 1 and reveal a new low-mass globular cluster candidate that we name VVV CL001.

Results. We use the horizontal-branch red clump to measure $E(B-V) \sim 2.2 \mathrm{mag},(m-M)_{0}=16.01 \mathrm{mag}$, and $D=15.9 \mathrm{kpc}$ for the globular cluster UKS 1. On the basis of near-infrared colour-magnitude diagrams, we also find that VVV CL001 has $E(B-V) \sim 2.0$, and that it is at least as metal-poor as UKS 1 , although its distance remains uncertain.

Conclusions. Our finding confirms the previous projection that the central region of the Milky Way harbours more globular clusters. VVV CL001 and UKS 1 are good candidates for a physical cluster binary, but follow-up observations are needed to decide if they are located at the same distance and have similar radial velocities.
\end{abstract}

Key words. globular clusters: general - globular clusters: individual: UKS 1 - globular clusters: individual: VVV CL001 - surveys

\section{Introduction}

The inner regions of the Milky Way have been mapped extensively at all wavelengths. Nethertheless, it is unknown whether more distant globular clusters await discovery, hidden beyond the bulge, because of the high density of stellar sources and the large and inhomogeneous interstellar extinction. Near-IR surveys provide an invaluable resource in searching for these clusters. The 2MASS, for instance, discovered two new globular clusters (Hurt et al. 2000). But its limiting magnitude of $K_{\mathrm{S}}=14.3$ (for $10 \sigma$-detections, Skrutskie et al. 2006) may prevent the discovery of fainter objects, especially if they are located in highly reddened regions.

The asymmetry of the spatial distribution of known globular clusters around the Galactic centre indicates that previous observations may have overlooked some additional globular clusters. Ivanov et al. (2005) estimated that there may be about ten clusters missing towards the inner Milky Way. Discoveries in the last ten years include both faint (low mass) halo clusters, as well as reddened globular clusters projected toward the bulge, e.g. 2MASS GC01 and 2MASS GC02 by Hurt et al. (2000) (see also Ivanov et al. 2000), ESO 280 SC06 by Ortolani et al. (2000), GLIMPSE C01 by Kobulnicky et al. (2005; but see also Ivanov et al. 2005; Davies et al. 2010), GLIMPSE C02 by Kurtev et al. (2008), AL-3 by Ortolani et al. (2006), FSR 1735 by Froebrich et al. (2007), Koposov 1 and Koposov 2 by Koposov et al. (2007), FSR 1767 by Bonatto et al. (2007), Whiting 1 (Carraro 2005), and Pfleiderer 2 by Ortolani et al. (2009).

The VISTA Variables in the Via Lactea (VVV) public survey has started mapping the inner disk and bulge of our Galaxy with the VISTA 4m telescope (Visible and Infrared Survey Telescope for Astronomy) in the near-IR (Minniti et al. 2010; Saito et al. 2010). One of the main scientific goals of the VVV survey is to study the bulge globular clusters and to search for new clusters. 

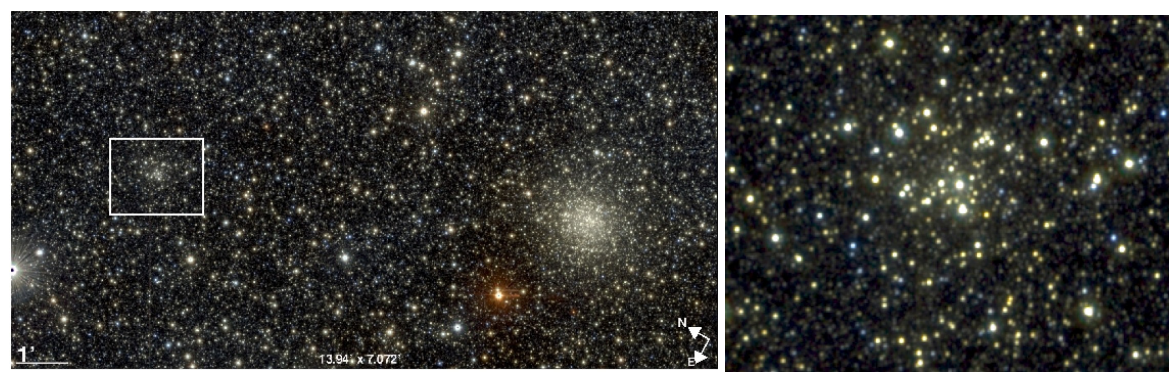

Fig. 1. Discovery image (left panel, $J H K_{\mathrm{S}}$ colour composite) showing VVV CL001 on the left and UKS 1 on the right. The cluster UKS 1 was discovered by Malkan et al. (1980), to have a total magnitude $I<16 \mathrm{mag}$, and was invisible in Palomar Survey plates (up to a few years ago, UKS 1 was known as "the faintest globular cluster of the Milky Way"). The right panel shows a zoom into the region centered on VVV CL001, corresponding to the white box $\left(1\right.$ '. $\left.^{\prime} 5 \times 11^{\prime} .5\right)$.

Here we present VVV CL001, the first globular cluster candidate discovered by the VVV survey.

\section{The VVV survey data}

The VVV survey data are acquired with the VISTA $4 \mathrm{~m}$ telescope at ESO Paranal Observatory (Emerson \& Sutherland 2010). The VVV field b351 was observed in the $J H K_{\mathrm{S}}$ bands under subarcsec seeing conditions $\left(0.8 \operatorname{arcsec}\right.$ in $\left.K_{\mathrm{S}}\right)$. The $Y Z$-band observations are still pending. Each one of the VVV fields (tiles) covers $1.636 \mathrm{deg}^{2}$ in total $\left(1.475^{\circ}\right.$ in $l$ by $1.109^{\circ}$ in $\left.b\right)$. The bulge field b351 observed here is centered at $\alpha=17: 50: 05.42$, $\delta=-23: 43: 16.7, l=4.987^{\circ}, b=1.838^{\circ}$.

We use here the images processed by the CASU VIRCAM pipeline v1.0 (e.g. Irwin et al. 2004). The photometry was obtained with DoPhot (Schechter et al. 1993). In addition, the photometry is uniformly calibrated against the 2MASS catalog (Skrutskie et al. 2006). The limiting magnitude of the single epoch VVV images is $K s=18.1$ in the bulge fields (for details of the observing strategy, see Minniti et al. 2010). The distance probed along the line of sight depends on the reddening of the fields. For example, in zero-reddening disk fields we would see horizontal-branch red clump beyond $50 \mathrm{kpc}$. Thus, we can search for distant galactic globular clusters and measure their physical parameters.

Visual inspection of the images of the field b351 led to the serendipitous discovery of a star cluster candidate that we name VVV CL001. This object is located in the vicinity of the known globular cluster UKS 1 (Fig. 1). On the basis of near-infrared stellar density maps (see Fig. 2), we conclude that this is not a statistical fluctuation of the background, and that the cluster VVV CL001 is centered at $\alpha=17: 54: 42.5, \delta=-24: 00: 53$ $\left(l=5.27^{\circ}, b=0.78^{\circ}\right)$. Applying the procedure developed by Koposov et al. (2008) and Belokurov et al. (2009), the statistical significance of the overdensity at the coordinates given above is $S=9.31$. There is no source identified at this location based on radio, infrared, and visible data from the SIMBAD, 2MASS and NED databases, or any other star cluster catalog. The second obvious density peak at $\alpha=17: 54: 38, \delta=-24: 00: 49$ is due to a saturated star.

The proximity of VVV CLO01 and UKS 1 on the sky (Fig. 1) made it appropriate to use the latter cluster as a comparison when measuring the reddening and distance of the new globular cluster candidate. We therefore concentrate first on measuring the parameters of UKS 1 using VVV Survey data.

\section{The globular cluster UKS 1}

Previous infrared photometry and spectroscopy of UKS 1 giants revealed that it is a very distant and reddened cluster, with
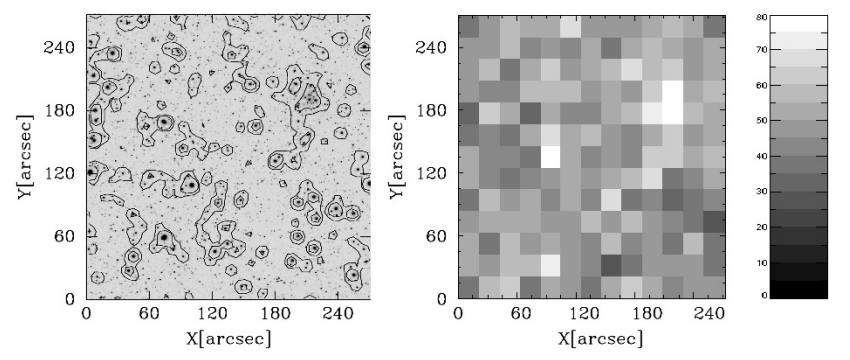

Fig. 2. $J$-band image of the VVV CL001 region (left) and density of all objects. The contours in the left panel refer to the intensity level of the image. In contrast, to derive the significance of the overdensity (right) only detections with a Dophot classification parameter 1 or 7 (Schechter et al. 1993) were selected. The sources were binned into 60× 60 pixel bins ( 20 by 20 arcsec). We note that even with PSF photometry we are unable to resolve the inner region of VVV CL001 (or UKS 1), which certainly affects the numerical results of our analysis (density, significance of the detected over-density). Both plots show the same 272 by 272 arcsec section of the $J$-band image.

distance modulus $\approx 15$, and $E(B-V) \approx 3$ (Minniti et al. 1995), and moderately metal-rich, with $[\mathrm{Fe} / \mathrm{H}]=-0.78$ (Origlia et al. 2005).

To determine the distance to UKS 1, we use the horizontalbranch red clump, which is very conspicuous for this cluster. The red clump stars with known parallaxes measured by the Hipparcos satellite are well-calibrated standard candles (Paczynski \& Stanek 1998; Alves 2000). Alves (2000) obtained a $K$-band calibration of the horizontal-branch red clump luminosity, and applied the calibration to the red clump of the Galactic bulge. For example, this calibration has also been applied to the red clump of the Large Magellanic Cloud (e.g., Alves et al. 2002; Pietrzyński et al. 2003a; Borissova et al. 2009). The uncertainties in the reddening should in all cases be larger than the uncertainties due to the unknown metallicity. Our $K_{\mathrm{S}}$ magnitudes are in the 2MASS magnitude system, hence we transform the $K$-band magnitudes of the red clump stars, studied by Alves (2000) to $K_{\mathrm{S}}$ magnitudes using $K=K_{\mathrm{S}}+0.044$ (from Grocholski $\&$ Sarajedini 2002). The zero-point differences should be smaller than 0.02 mag (Alves et al. 2002). From the previously cited works, we adopt the following mean values for the red clump stars: $M_{K_{\mathrm{s}}}=-1.65 \pm 0.03$, and $\left(J-K_{\mathrm{s}}\right)_{0}=0.71 \pm 0.03$ (López-Corredoira et al. 2002). The distance modulus to the horizontal-branch red clump in the globular cluster would be:

$\mu=m_{K_{\mathrm{s}}}-A_{K_{\mathrm{s}}} /\left(A_{J}-A_{K_{\mathrm{s}}}\right)\left[\left(J-K_{\mathrm{s}}\right)-\left(J-K_{\mathrm{s}}\right)_{0}\right]-M_{K_{\mathrm{s}}}$.

Adopting the mean red-clump magnitudes and colours discussed above, and the reddening coefficients from Cardelli et al. (1989), 


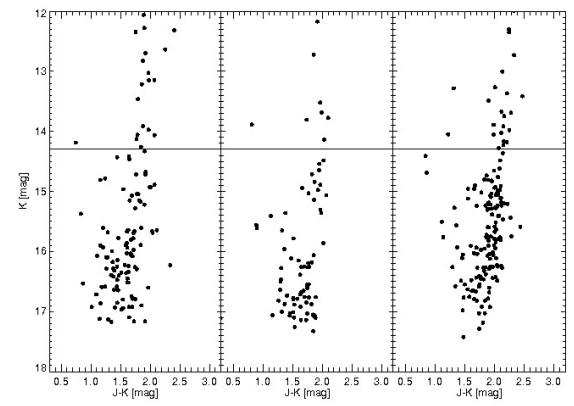

Fig. 3. Discovery near-infrared colour-magnitude diagram of VVV CL001 for a 15 arcsec radius area centered on the cluster (left) compared with a field of the same area located 3.75 east (middle), and a field of UKS 1 of the same size that is offset from the cluster centre by $\left(45^{\prime \prime} .0\right)$ (right). The small offset for UKS 1 was applied, since the centre of this cluster is barely resolved. The horizontal line at $K_{\mathrm{s}}=14.3$ shows the limit of 2MASS photometry. Comparing UKS 1 and VVV CL001, we find that the RGB of UKS 1 is much more populated, hence VVV CL001 is far less massive then UKS 1.

this simplifies to

$\mu=5 \log d(\mathrm{pc})-5=m_{K_{\mathrm{s}}}-0.73\left(J-K_{\mathrm{s}}\right)+2.17$.

Using this equation, we compute the mean distance modulus (and distance in $\mathrm{kpc}$ ) for the horizontal-branch red clump in UKS 1 , which has mean values of $K_{\mathrm{S}}=15.3$ and $J-K_{\mathrm{S}}=2.0$ (Fig. 4). This yields a mean $E(J-K)=1.30$, equivalent to $E(B-V)=2.5$, and a distance modulus $(m-M)_{0}=16.01$, equivalent to $15.9 \mathrm{kpc}$. The uncertainty in the red-clump position was estimated to be $\Delta m_{K_{\mathrm{S}}}=0.012 \mathrm{mag}$ and $\Delta\left(J-K_{\mathrm{S}}\right)=0.08 \mathrm{mag}$, based on analysis of the relevant region of the CMD. This results in an error in the distance modulus and corresponding distance of $\Delta \mu=0.078$ and $\Delta D=0.6 \mathrm{kpc}$. The distance to UKS 1 was also measured by Ortolani et al. (2007) using deep CST photometry. They found the mean magnitude of the horizontal branch to be $J=17.69$, and the mean colour $J-H=1.39 \mathrm{mag}$ or $1.71 \mathrm{mag}$ depending on the calibration, and estimated a distance of between $D=9.3-14.3 \mathrm{kpc}$, placing it beyond the Galactic centre. They inferred a total reddening of $E(B-V)=2.94-3.59 \mathrm{mag}$ also depending on the calibration. Our values are consistent with the lower reddening values of Ortolani et al. (2007). However, our distance measurement favours the largest distance measurement of Ortolani et al. (2007), and is consistent with UKS 1 being located well beyond the Galactic centre, but not as far as the Sgr dwarf galaxy discovered by Ibata et al. (1994).

\section{The globular cluster candidate VVV CL001}

Figure 3 shows that the red giant branch of VVV CL001 is well defined, but the precise location of the horizontal-branch red clump is not, and we cannot use the same method to estimate the distance. However, Fig. 4 shows that the RGB of VVV CL001 is bluer than that of UKS 1 . This may be due to either a lower extinction, a lower metallicity, or a combination of both effects. Within the selected sample, the saturation of the brightest RGB stars prevents us from using the tip of the RGB for an independent distance determination. The comparison between red clump and tip of the RGB distance estimates could otherwise be used to assess the accuracy of the distance. Although the absence of a populated horizontal branch red clump in VVV CL001 may be due to low metallicity, we test the possibility that this is a statistical effect caused by the small sample. In addition, the

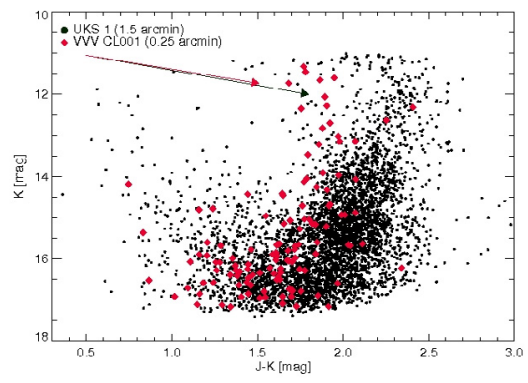

Fig. 4. CMD of VVV CL001 (diamonds) compared with UKS 1 (dots). The horizontal-branch red clump of UKS 1 is seen at $K_{\mathrm{S}}=15.3$, $J-K_{\mathrm{S}}=2.0$. This diagram shows that the RGB of VVV CL001 is slightly bluer than that of UKS 1 , from which one can conclude that VVV CL001 is less reddened or more metal-poor than UKS 1, and that the RGB of UKS 1 is much more populated, indicative of the low mass of VVV CL001. The legend gives the radius of the selected cluster region, whereas the arrows indicate the reddening derived for UKS 1.

CMDs of a randomly selected UKS 1 stars corresponding to the VVV CL001 sample size do not display a distinct HB. This leads us to the conclusion that the missing HB is mostly a statistical effect. Refining the selection criteria and a higher spatial resolution may help us to detect some HB stars.

Based on the CMD and assuming similar reddenings for both clusters, we can conclude that VVV CL001 has a similar metallicity or is slightly more metal-poor than UKS 1 . It would otherwise be hard to reconcile the clearly different colours of the RGBs of these two clusters (see Fig. 3), particularly since a large difference in metallicity is impossible. The integrated near-infrared luminosity for Milky Way globular clusters are not very well known (Cohen et al. 2007, and references therein), nevertheless by comparing the RGB population in VVV CL001 and UKS 1 we also estimate that the new cluster candidate is $\approx 3.7$ mag fainter in $K_{\mathrm{S}}$ than UKS 1 .

Thus, even though we cannot determine its distance accurately, the comparison with UKS 1 allows us to conclude that VVV CL001 is located beyond the Galactic centre, on the opposite side of the Milky Way. Therefore, this does not appear to be a bulge globular cluster according to the definitions of Minniti et al. (1995) and Barbuy et al. (1998).

For consistency, we can also determine the reddening from the mean location of the VVV CL001 red giants in the $J-H$ versus $H-K_{\mathrm{S}}$ colour-colour diagram. The mean $J-H=1.4$ and $H-K_{\mathrm{S}}=0.4$ yield $A_{\mathrm{V}}=6 \mathrm{mag}$ and $E(B-V)=2.0$, which are slightly less reddened than UKS 1 . On the other hand, the maps of Schlegel et al. (1998) give a mean reddening of $E(B-V) \approx$ 3.3 for this region of VVV field b351, which is larger than our measurements, but the reddening towards the bulge is known to be non-uniform and patchy.

The data are not good enough to measure the distance, but we have an accurate reddening and location, and deeper followup observations would allow us to define the other structural parameters of VVV CL001. On the basis of the number of giants, we crudely estimate that this cluster has a low mass, being about 50-100 times less massive than UKS 1. In that case, VVV CL001 would join the list of very low-luminosity globular clusters in the Milky Way (Table 1, see also Koposov et al. 2007). Given the uncertainty in the cluster distance, the possibility of VVV CL001 being an old, compact open cluster is at this point not strictly ruled out, although the high stellar concentration and the well populated RGB favour the globular cluster interpretation. 
Table 1. Properties of VVV CL001, based on the VVV data, compared to two known extremely low luminosity globular clusters Koposov 1 and 2 (Koposov et al. 2007).

\begin{tabular}{lrrr}
\hline \hline & VVV CL001 & Koposov 1 & Koposov 2 \\
Position $[l, b]$ & $4.99^{\circ}, 1.84^{\circ}$ & $260.98^{\circ}, 70.75^{\circ}$ & $195.11^{\circ}, 25.55^{\circ}$ \\
Distance [kpc] & uncertain & $\approx 50 \mathrm{kpc}$ & $\approx 40 \mathrm{kpc}$ \\
Radius [arcmin] & $\approx 1 \mathrm{pc}^{*}$ & $\approx 3 \mathrm{pc}$ & $\approx 3 \mathrm{pc}$ \\
\hline
\end{tabular}

Notes. ${ }^{(*)}$ Based on a distance of $16 \mathrm{kpc}$ and a selected radius of 15 arcsec.

The proximity of these two globular clusters in the sky is surprising. There are no other globular cluster pairs in the Milky Way separated by only 8 arcmin. If they are located at the same distance, and have the same radial velocity, these two clusters may be bound: assuming the distance of UKS 1 to be $15.9 \pm 0.6 \mathrm{kpc}$, the separation would be less than $40 \mathrm{pc}$. There are physical pairs of clusters known in other galaxies (e.g., Bhatia \& Hatzidimitriou 1988; Dieball et al. 2002; Bekki et al. 2004; Minniti et al. 2004). However, the present initial results are not good enough to indicate that VVV CL001 is at the same distance as UKS 1, and therefore we cannot claim that they form a physical binary cluster. We still need to measure their radial velocities to test the binary nature of the two clusters.

Alternatively, this may be just a chance alignment, and VVV CL001 could be a globular cluster or, although less likely, an old open cluster (see Sect. 4), either more distant or closer to the Sun than UKS 1. Deeper photometric observations are also clearly needed for this object.

\section{Conclusions}

The VVV survey is searching for missing globular clusters in the inner regions of the Milky Way galaxy. We have reported the discovery of VVV CL001 (Fig. 1), a low-mass globular cluster candidate at $\alpha=17: 54: 42.5, \delta=-24: 00: 53$. This is located only 8 arcmin away from the Galactic globular cluster UKS 1 in the sky. We have taken advantage of this spatial proximity to use UKS 1 as reference in order to estimate the parameters of this newly discovered cluster. We have measured the distance and reddening of UKS 1 , finding $E(B-V)_{\mathrm{UKS} 1}=2.5$ and $D_{\mathrm{UKS} 1}=$ $15.9 \mathrm{kpc}$.

We have presented the first colour-magnitude diagrams of the new cluster VVV CL001 (Fig. 3), estimating $E(B-V)=2.0$. We have been unable to define the mean $K_{S}$-band magnitude of the horizontal-branch red clump for this cluster, hence and its distance remains uncertain. A comparison with the RGB of UKS 1 has provided a very crude estimate that it is at a similar distance, placing VVV CL001 well beyond the bulge of the Milky Way. Observations in the $K_{\mathrm{S}}$-band that will be acquired in the future seasons of the VVV survey would allow us to improve this CMD of Fig. 3 and define the HB as well as the MSTO, which would allow a photometric age determination. In addition, we estimate that the RR Lyrae of VVV CL001 would be within the limit of detection of our VVV Survey.

We have been unable to definitely conclude that the proximity of UKS 1 and VVV CL001 on the sky imply that they represent a binary cluster because the distance to VVV CL001 is too uncertain. However, that they are a binary remains an exciting possibility that needs to be confirmed not only by means of a more accurate distance determination, but also by measuring their respective radial velocities from spectroscopic measurements. Finally, the present results are very encouraging and we conclude that the VVV survey can potentially provide the largest and most homogeneous census of globular clusters in the survey area, out to well beyond the Galactic centre, even in regions of large extinction.

Acknowledgements. We thank the Cambridge Astronomical Survey Unit (CASU) for processing the VISTA raw data. We acknowledge support by the FONDAP Center for Astrophysics 15010003, BASAL Center for Astrophysics and Associated Technologies PFB-06, MILENIO Milky Way Millennium Nucleus P07-021-F from MIDEPLAN, FONDECYT 1090213 from CONICYT, and the European Southern Observatory. We use data products from the Two Micron All Sky Survey, which is a joint project of the University of Massachusetts and the Infrared Processing and Analysis Center/California Institute of Technology, funded by the National Aeronautics and Space Administration and the National Science Foundation. We also thank the referee, whose comments helped to improve the paper significantly.

\section{References}

Alves, D. R. 2000, ApJ, 539, 732

Alves, D. R., Rejkuba, M., Minniti, D., \& Cook, K. H. 2002, ApJ, 573, L51

Barbuy, B., Bica, E., \& Ortolani, S. 1998, A\&A, 333, 117

Bekki, K., Beasley, M. A., Forbes, D. A., \& Couch, W. J. 2004, ApJ, 602, 730 Belokurov, V., Zucker, D. B., Evans, N. W., et al. 2007, ApJ, 654, 897 Belokurov, V., Walker, M. G., Evans, N. W., et al. 2009, MNRAS, 397, 1748 Bhatia, R. K., \& Hatzidimitriou, D. 1988, MNRAS, 230, 215

Bonatto, C., Bica, E., Ortolani, S., \& Barbuy, B. 2007, MNRAS, 381, L45

Borissova, J., Rejkuba, M., Minniti, D., Catelan, M., \& Ivanov, V. D. 2009, A\&A, 502, 505

Cardelli, J. A., Clayton, G. C., \& Mathis, J. S. 1989, ApJ, 345, 245

Carraro, G. 2005, ApJ, 621, L61

Cohen, J. G., Hsieh, S., Metchev, S., Djorgovski, S. G., \& Malkan, M. 2007, AJ, 133, 99

Davies, B., Bastian, N., Gieles, M., et al. 2011, MNRAS, in press [arXiv: 1010.2973]

Dieball, A., Müller, H., \& Grebel, E. K. 2002, A\&A, 391, 547

Emerson, J., \& Sutherland, W. 2010, The Messenger, 139, 2

Froebrich, D., Meusinger, H., \& Scholz, A. 2007, MNRAS, 377, L54

Grocholski, A. J., \& Sarajedini, A. 2002, AJ, 123, 1603

Harris, W. E. 1996, AJ, 112, 1487

Hurt, R. L., Jarrett, T. H., Kirkpatrick, J. D., et al. 2000, AJ, 120, 1876

Ibata, R. A., Gilmore, G., \& Irwin, M. J. 1994, Nature, 370, 194

Irwin, M. J., Lewis, J., Hodgkin, S., et al. 2004, in Society of Photo-Optical Instrumentation Engineers (SPIE) Conference Series, Society of PhotoOptical Instrumentation Engineers (SPIE) Conf. Ser., ed. P. J. Quinn, \& A. Bridger, 5493, 411

Ivanov, V. D., Borissova, J., \& Vanzi, L. 2000, A\&A, 362, L1 Ivanov, V. D., Kurtev, R., \& Borissova, J. 2005, A\&A, 442, 195 Kobulnicky, H. A., Monson, A. J., Buckalew, B. A., et al. 2005, AJ, 129, 239 Koposov, S., de Jong, J. T. A., Belokurov, V., et al. 2007, ApJ, 669, 337 Koposov, S., Belokurov, V., Evans, N. W., et al. 2008, ApJ, 686, 279

Kurtev, R., Ivanov, V. D., Borissova, J., \& Ortolani, S. 2008, A\&A, 489, 583

López-Corredoira, M., Cabrera-Lavers, A., Garzón, F., \& Hammersley, P. L. 2002, A\&A, 394, 883

Malkan, M., Kleinmann, D. E., \& Apt, J. 1980, ApJ, 237, 432

Minniti, D., Olszewski, E. W., \& Rieke, M. 1995, AJ, 110, 1686

Minniti, D., Rejkuba, M., Funes, J. G., \& Kennicutt, Jr., R. C. 2004, ApJ, 612, 215

Minniti, D., Lucas, P. W., Emerson, J. P., et al. 2010, New A, 15, 433

Origlia, L., Valenti, E., Rich, R. M., \& Ferraro, F. R. 2005, MNRAS, 363, 897

Ortolani, S., Bica, E., \& Barbuy, B. 2000, A\&A, 361, L57

Ortolani, S., Bica, E., \& Barbuy, B. 2006, ApJ, 646, L115

Ortolani, S., Barbuy, B., Bica, E., Zoccali, M., \& Renzini, A. 2007, A\&A, 470, 1043

Ortolani, S., Bonatto, C., Bica, E., \& Barbuy, B. 2009, AJ, 138, 889

Paczynski, B., \& Stanek, K. Z. 1998, ApJ, 494, L219

Pietrzyński, G., Gieren, W., \& Udalski, A. 2003a, AJ, 125, 2494

Pietrzyński, G., Gieren, W., \& Udalski, A. 2003b, AJ, 125, 2494

Saito, R., Hempel, M., Alonso-García, J., et al. 2010, The Messenger, 141, 24

Schechter, P. L., Mateo, M., \& Saha, A. 1993, PASP, 105, 1342

Schlegel, D. J., Finkbeiner, D. P., \& Davis, M. 1998, ApJ, 500, 525

Skrutskie, M. F., Cutri, R. M., Stiening, R., et al. 2006, AJ, 131, 1163 